\title{
Performance of CT ASPECTS and Collateral Score in Risk Stratification: Can Target Perfusion Profiles Be Predicted without Perfusion Imaging?
}

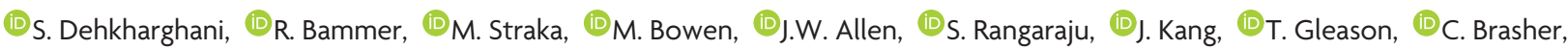 \\ and (1) F. Nahab
}

\begin{abstract}
BACKGROUND AND PURPOSE: Endovascular trials suggest that revascularization benefits a subset of acute ischemic stroke patients with large-artery occlusion and small-core infarct volumes. The objective of our study was to identify thresholds of noncontrast CT-ASPECTS and collateral scores on CT angiography that best predict ischemic core volume thresholds quantified by $\mathrm{CT}$ perfusion among patients with acute ischemic stroke.
\end{abstract}

MATERIALS AND METHODS: Fifty-four patients with acute ischemic stroke ( $<12$ hours) and MCA/intracranial ICA occlusion underwent NCCT/CTP during their initial evaluation. CTP analysis was performed on a user-independent platform (RApid processing of Perfuslon and Diffusion), computing core infarct (defined as CBF of $<30 \%$ normal). A target mismatch profile consisting of infarction core of $\leq 50 \mathrm{~mL}$ was selected to define candidates with acute ischemic stroke likely to benefit from revascularization.

RESULTS: NCCT-ASPECTS of $\geq 9$ with a CTA collateral score of 3 had $100 \%$ specificity for identifying patients with a CBF core volume of $\leq 50 \mathrm{~mL}$. NCCT-ASPECTS of $\leq 6$ had $100 \%$ specificity for identifying patients with a CBF core volume of $>50 \mathrm{~mL}$. In our cohort, 44 ( $81 \%$ ) patients had an NCCT-ASPECTS of $\geq 9$, a CTA collateral score of 3 , or an NCCT-ASPECTS of $\leq 6$.

CONCLUSIONS: Using an NCCT-ASPECTS of $\geq 9$ or a CTA collateral score of 3 best predicts CBF core volume infarct of $\leq 50 \mathrm{~mL}$, while an NCCT-ASPECTS of $\leq 6$ best predicts a CBF core volume infarct of $>50 \mathrm{~mL}$. Together these thresholds suggest that a specific population of patients with acute ischemic stroke not meeting such profiles may benefit most from CTP imaging to determine candidacy for revascularization.

ABBREVIATIONS: AIS = acute ischemic stroke; RAPID = RApid processing of Perfuslon and Diffusion

$\mathbf{R}$ evascularization aims to prevent progression of ischemic injury in acute ischemic stroke (AIS). ${ }^{1-4}$ Recent success in trials of endovascular AIS therapy, while restoring motivation for acute stroke intervention, has left the subject of an optimal patient-selection paradigm largely unaddressed. ${ }^{5-8}$ While the primary goals in this setting include timely revascularization, the relative merits of expedited triage versus identification of target imaging profiles remain the subject of ongoing inquiry.

Received November 20, 2015; accepted after revision January 10, 2016

From the Departments of Radiology and Imaging Sciences (S.D., M.B., J.W.A., T.G.) and Neurology (S.D., J.W.A., S.R., C.B., F.N.), Emory University Hospital, Atlanta, Georgia; Department of Radiology (R.B.), Stanford University Hospital, Stanford, California; Institut für Radiologie und Nuklearmedizin (M.S.), Kantonsspital Winterthur, Winterthur, Switzerland; and Department of Biostatistics (J.K.), University of Michigan, Ann Arbor, Michigan.

Please address correspondence to Seena Dehkharghani, MD, Department of Radiology and Imaging Sciences and Neurology, Neuroradiology Division; Attention: Mary Davis, Department of Radiology and Imaging Sciences, Emory University Hospital, 1364 Clifton Rd NE, Atlanta, GA 30322; e-mail: Seena.Dehkharghani@Emory.edu

http://dx.doi.org/10.3174/ajnr.A4727
Contemporary guidelines on AIS management, therefore, remain inconclusive as to the role of multimodal imaging selection. ${ }^{9}$

We recently reported the benefits of a high-speed computing tool for CT perfusion analysis over qualitative approaches to imaging triage for prognostication among patients with anterior circulation AIS. ${ }^{10}$ The findings therein suggested that a user- and vendor-independent computational tool may outperform purely qualitative approaches in outcome prediction. Similar implementations of this tool in recent, prospective endovascular trials suggested strong results as an approach to patient selection; however, the relative contribution of CTP-based selection criteria, among other trial-specific features, remains uncertain in light of the overall favorable outcomes reported across disparate trial designs. ${ }^{5-8}$

The objective of our study was to identify thresholds of NCCTASPECTS and collateral score on CT angiography that best predict ischemic core volume thresholds quantified by CTP among patients with AIS.

AJNR Am J Neuroradiol 37:1399-404 Aug 2016 www.ajnr.org

1399 


\section{MATERIALS AND METHODS}

The population examined was previously studied in a report of the relative predictive value of quantitative and qualitative acute stroke imaging analysis in prognostication and clinical outcome prediction. ${ }^{10}$ Briefly, 62 continuous patients ( 36 women; median age, 70 years; range, 33-94 years) with AIS ( $<12$ hours) and MCA or intracranial ICA occlusion were identified from a prospectively collected, single-institution stroke registry and radiologic informatics query of 815 patients with ischemic stroke, spanning February 1, 2011, to December 31, 2013, with Emory University Hospital review board approval. All patients were evaluated initially by a dedicated vascular neurologist in the emergency setting, with initiation of institutional stroke protocol facilitating expedited triage, imaging, interpretation, and treatment when appropriate. All patients underwent comprehensive stroke imaging at presentation, including NCCT, CTA, and CTP. Patients were included in the analysis on the basis of successful completion of the imaging protocol, absence of motion or other artifacts rendering imaging nondiagnostic, and the absence of large hemorrhages such as parenchymal hematomas (types 1 and 2) potentially confounding final infarction measurement. Exclusion criteria were an inability to undergo multimodal CT, a history of renal failure, and patient age younger than 18 years. A subset of patients received intravenous and/or intra-arterial thrombolytic therapy as per institutional protocol and at the discretion of the treating vascular neurologist and neurointerventionalist.

\section{Imaging Protocol}

All patients underwent an institutional stroke imaging protocol including NCCT, CTP, and CTA. CT was performed on a $40-\mathrm{mm}$, 64-detector row clinical system (LightSpeed VCT; GE Healthcare, Milwaukee, Wisconsin). Helical NCCT (120 kV[peak], 100350 auto-mAs) was performed from the foramen magnum through the vertex at 5.0-mm section thickness. In the absence of visible intracranial hemorrhage during real-time evaluation by a radiologist and stroke neurologist, 2 contiguous CTP slabs were obtained for $8-\mathrm{cm}$ combined coverage of the supratentorial brain, obtained at 5-mm sections per slab. Cine mode acquisition (80 $\mathrm{kVp}, 100 \mathrm{mAs}$ ) permitting high-temporal-resolution (1-second sampling interval) dynamic bolus passage imaging was performed following the administration of $35 \mathrm{~mL}$ of iodinated contrast (iopamidol, Isovue 370; Bracco Diagnostics, Princeton, New Jersey), power injected at $5 \mathrm{~mL} / \mathrm{s}$ through an 18-ga or larger antecubital IV access. Contrast administration was followed by a $25-\mathrm{mL}$ saline flush at the same rate. For both slabs, the same acquisition and injection protocol was used (ie, a total of $70 \mathrm{~mL}$ of iodine was used for CTP). Last, helical CTA (120 kVp, 200-350 auto-mAs) was performed from the carina to the vertex (section thickness/interval, $0.625 / 0.375 \mathrm{~mm}$ ) following IV administration of $70 \mathrm{~mL}$ of iodinated contrast injected at $5 \mathrm{~mL} / \mathrm{s}$ and followed by a $25-\mathrm{mL}$ saline flush.

Follow-up imaging in all patients included brain MR imaging for documentation of final infarct size within 3 days of CTP, performed on a 3T (Tim Trio; Siemens, Erlangen, Germany) clinical whole-body system with local signal reception by a dedicated 12 channel head coil. All images were transferred to a separate workstation for analysis (Mac Pro; Apple, Cupertino, California) by using a third-party DICOM viewer (OsiriX Imaging Software; http:// www.osirix-viewer.com). The details of the postprocessing pipeline were reported previously. ${ }^{10}$

\section{Imaging Analysis}

NCCT-ASPECTS. ASPECTSs were assigned by 2 experienced vascular neurologists (S.R., F.N.) blinded to all other imaging and clinical outcomes. ASPECTS uses a 10-point visual inspection scale estimating ischemic burden in the supratentorial brain as detailed previously by Barber et al. ${ }^{11}$

CTA Collateral Score. A CTA-derived collateral vessel-scoring methodology was used as detailed previously. ${ }^{10,12}$ Briefly, 2 experienced neuroradiologists, both with subspecialty certification and experienced in stroke and neurovascular imaging, assigned CTA collateral scores using a visual inspection methodology to quantify surface leptomeningeal collaterals in response to proximal arterial compromise, compared with the contralateral side. ${ }^{12}$ The neuroradiologists assigned scores blinded to clinical and outcomes data and all other imaging. ${ }^{13,14}$ A collateral score was assigned by using an ordinal, visual grading system estimating collateral flow, scored $0-3$ as follows: Collateral flow was assigned a score of zero for absent surface vasculature, 1 for $>0$ but $\leq 50 \%$ vasculature, 2 for $>50$ but $<100 \%$ vasculature, and 3 for normal or supranormal surface vasculature of the MCA territory. CTA analysis was performed by using $20-\mathrm{mm}$ axial sliding maximum intensity projection and $0.625-\mathrm{mm}$ axial source images and orthogonal and curved multiplanar reformats as needed.

CT Perfusion. All perfusion imaging was postprocessed by using a custom, noncommercial version of a vendor-independent software platform (RAPID) provided by Stanford University. ${ }^{15}$ RAPID is an automated computational tool designed for timely analysis of CTP data as used recently in the stroke trial setting. ${ }^{3-5,7}$ Details of the perfusion postprocessing pipeline were discussed previously. ${ }^{10,15}$ Briefly, following preprocessing steps correcting rigid-body motion, arterial input function selection is performed and deconvolved from the voxel time-attenuation course using a delay-insensitive algorithm for isolation of the tissue residue function. The time to maximum of the tissue residue function is determined on a voxelwise basis, and time-to-maximum maps are incrementally thresholded between 4 and 10 seconds at 2-second intervals with penumbral maps overlaid on the source CTP data. ${ }^{10,15}$

Cerebral blood flow maps expressed in milliliters $/ 100 \mathrm{~g} / \mathrm{min}$ ute were computed as outlined elsewhere. Relative CBF maps have been used in the stroke trial setting as estimates of irreversibly infarcted (core) tissues by using thresholds of relative CBF of $<30 \%$ contralateral normal tissues. ${ }^{16}$ Parametric maps were automatically generated and overlaid on source images for review purposes.

\section{Statistical Analysis}

The range, mean, and median values of relative CBF-derived infarction core were determined across ASPECTS levels and for dichotomized ASPECTS of $\geq 7$. A linear regression model was fitted with relative CBF core volume as an outcome, creating dummy variables for covariate NCCT-ASPECTS in regression analysis; 
Table 1: Patient characteristics ${ }^{\mathrm{a}}$

\begin{tabular}{lc}
\hline \multicolumn{1}{c}{ Characteristics } \\
\hline Admission NIHSS & $15(16)$ \\
Time of onset/last known healthy to imaging (min) & $210(252)$ \\
IV tPA (No.) (\%) & $23(43)$ \\
Endovascular treatment (No.) (\%) & $9(17)$ \\
$\quad$ IA tPA & 3 \\
Thrombectomy & 6 \\
NCCT-ASPECTS & $9(1)$ \\
Final infarction volume $(\mathrm{mL})$ & $37(96)$ \\
\hline
\end{tabular}

Note:-IA indicates intra-arterial.

${ }^{a}$ Data are reported as median (interquartile range) unless otherwise noted.

ANOVA analysis was performed on the fitted linear regression, and an F-test was applied. Because the NCCT-ASPECTS was considered a dummy variable in the regression and ANOVA analyses, the Kendall $\tau$ correlation was used to test the strength of the correlation. Given the previously high interreader correlation for the NCCT-ASPECTS of $\geq 7$ (0.93) in our study population, all variables were assessed as the unweighted mean of combined reader scores for qualitative variables. ${ }^{10}$

An operationally defined CTP profile predicting favorable outcome was assigned as prescribed in a recently reported, prospective endovascular therapy trial. ${ }^{5}$ Specifically, a target mismatch profile consisting of an infarction core of $\leq 50 \mathrm{~mL}$ was selected as the target relative $\mathrm{CBF}$ infarction core volume determined across NCCT-ASPECTS and at a dichotomized NCCTASPECTS of $\geq 7$. The $50 \mathrm{~mL}$ threshold was selected as a reference volume as reported by the investigators of the recent Solitaire With the Intention for Thrombectomy as Primary Endovascular Treatment (SWIFT PRIME) trial and as used in the recent development of a benchmarking software environment for quality control in stroke perfusion imaging. ${ }^{5,17}$ The Fisher exact test was further applied to determine the association of ASPECTS of $\geq 7$ and infarction core of $\leq 50 \mathrm{~mL}$. A prediction error model for correct classification of patients as having greater or less than 50 $\mathrm{mL}$ CBF infarction core was determined across NCCT-ASPECTS and at a dichotomized ASPECTS of $\geq 7$. Sensitivity, specificity, and positive and negative predictive values for various thresholds of ASPECTS and collateral scores were calculated and reported.

Statistical analysis was performed in R statistical and computing software (http://www.r-project.org/).

\section{RESULTS}

Sixty-two patients (36 women; median age, 70 years; range, 33-94 years) with AIS ( $<12$ hours) and MCA or intracranial ICA occlusion constituted the study population as previously reported. Incomplete or degraded imaging necessitated exclusion of 8 patients, leaving 54 patients for analysis. An ICA or M1 occlusion was present in $41(76 \%)$ patients, with proximal M2 segment occlusion in the remainder. No patients had bilateral arterial occlusions.

As shown in Table 1, the median NIHSS score at admission was 15 (interquartile range, 16); the mean duration from the time of onset/last known healthy to imaging was 210 minutes. Twentythree $(43 \%)$ patients received IV tPA, and $9(17 \%)$ underwent endovascular treatment with intra-arterial tPA $(n=3)$ or thrombectomy $(n=6)$. The median NCCT-ASPECTS was 9 (interquar-

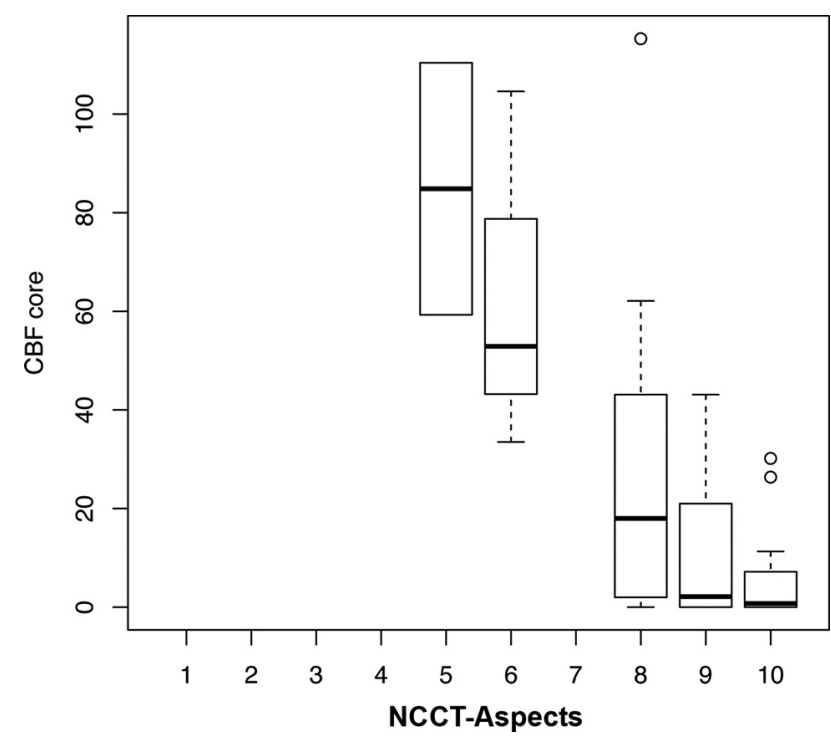

FIG 1. Computed ischemic core volumes by using relative CBF thresholded at $<30 \%$ contralateral normal tissues. Boxplots illustrate the range and distribution of ischemic core values across NCCT-ASPECTS values for the entire study population. An ASPECTS of 7 represented a null dataset following averaging of 2 blinded readers (see text). Notably, no patients with NCCT-ASPECTS of $\leq 5$ were encountered within the study population. Values are shown as median (line), interquartile range (box), 10th/90th percentile (bars), and outliers (circles).

tile range, 1). Median final infarction volume as measured by MR imaging was 37 (interquartile range, 96) $\mathrm{mL}$.

While a significant association was detected between CBF core estimates and NCCT-ASPECTS (Kendall $\tau$ correlation, -0.51 ; $P<.01$ ), large variability was found across 2-reader mean ASPECTS values (Fig 1). For example, across patients with an NCCT-ASPECTS of 8, the CBF core volume ranged from 0 to 115 $\mathrm{mL}$ with a median of $23 \mathrm{~mL}$ (interquartile range, $42 \mathrm{~mL}$ ). Ranges of core volume increased further at lower ASPECTS. Table 2 presents sensitivity, specificity, negative predictive values, and positive predictive values of the candidate predictors, NCCT-ASPECTS and collateral scores. For the analysis of dichotomized ASPECTS of $\geq 7$, the CBF core volume ranged from 0 to $115 \mathrm{~mL}$ with a median of $4 \mathrm{~mL}$ (interquartile range, $15 \mathrm{~mL}$ ). An NCCTASPECTS of $\geq 9$ had $100 \%$ specificity ( $95 \%$ CI, 60-100) for identifying patients with $\mathrm{CBF}$ core volume of $\leq 50 \mathrm{~mL}$, while an NCCT-ASPECTS of $\leq 6$ had $100 \%$ specificity (95\% CI, $90 \%-$ $100 \%$ ) for identifying patients with a CBF core volume of $>50$ $\mathrm{mL}$. The prediction error model for correct identification of infarction core of $\leq 50 \mathrm{~mL}$ among ASPECTS of $\geq 7$ demonstrated significant associations but low specificity relative to a CBF core of $\leq 50 \mathrm{~mL}$ (prediction error, $9 \% ; P=.025$; sensitivity, 0.98 ; specificity, 0.50 ; negative predictive value, 0.80 ; positive predictive value, 0.92 ).

Our cohort included 28 patients with a collateral score of 1, 10 patients with a score of 2 , and 15 patients with a score of 3 . A collateral score of 3 on CTA had $100 \%$ specificity (95\% CI, 47\%$99 \%$ ) but only $33 \%$ sensitivity ( $95 \%$ CI, $20 \%-49 \%$ ) for identifying patients with AIS with a CBF core volume of $\leq 50 \mathrm{~mL}$. A collateral score of $\leq 1$ had an $88 \%$ sensitivity (95\% CI, 47\%-99\%) and a $53 \%$ specificity $(95 \%$ CI, $38 \%-68 \%$ ) for identifying patients with AIS with a CBF core volume of $>50 \mathrm{~mL}$. The collateral 
score demonstrated a significant association with CBF ischemic core volumes $(P<.01)$.

\section{DISCUSSION}

Our study found significant variability in CBF core volumes among patients with AIS with similar NCCT-ASPECTS, we identified thresholds of NCCT-ASPECTS of $\geq 9$ and collateral scores of 3 with high specificity for finding patients with AIS with core volumes considered ideal for revascularization. We also found that NCCT-ASPECTS of $\leq 6$ had a high specificity for identifying patients with AIS with core volumes that made them suboptimal candidates for revascularization.

Recent successful AIS trials have used disparate methodologies for patient selection. These have differed primarily in their use of either fast but potentially insensitive methodologies (eg, NCCTASPECTS) versus more rigorous approaches to estimating tissue viability with CTP, permitting operational tissue classification,

Table 2: Accuracy of NCCT-ASPECTS and collateral score in prediction of CBF infarction core of $\leq 50 \mathrm{~mL}$

\begin{tabular}{lcccc}
\hline \multicolumn{1}{c}{ Threshold } & Sensitivity & Specificity & PPV & NPV \\
\hline NCCT-ASPECTS (CBF core volume $\leq 50 \mathrm{~mL})$ & & & & \\
$\geq 4$ & 100 & 0 & 84.9 & - \\
$\geq 5$ & 100 & 25 & 88.2 & 100 \\
$\geq 6$ & 100 & 37.5 & 90.0 & 100 \\
$\geq 7$ & 97.8 & 50 & 91.7 & 80.2 \\
$\geq 8$ & 91.1 & 62.5 & 93.2 & 55.5 \\
$\geq 9$ & 68.9 & 100 & 100 & 36.4 \\
$\geq 10$ & 26.7 & 100 & 100 & 19.5 \\
Collateral score (CBF core volume $\leq 50 \mathrm{~mL})$ & & & & \\
$\geq 0$ & 100 & 0 & 84.9 & - \\
$\geq 1$ & 53.3 & 87.5 & 96.0 & 25.0 \\
$\geq 2$ & 33.3 & 100 & 100 & 21.0 \\
$\geq 3$ & 0 & 100 & - & 15.1 \\
\hline
\end{tabular}

Note:-PPV indicates positive predictive value; NPV, negative predictive value; -, non-value. segmentation, and volume measures. ${ }^{3,4,7,18}$ These specific factors have been emphasized as targets for optimization and general requirements in the stroke-research setting in recent expert consensus. ${ }^{19}$ NCCT-ASPECTS aims to qualitatively identify early ischemic changes modulated by ischemic bulk water shifts (ie, edema). The speed and nearly invariable access to NCCTASPECTS are clearly advantageous; however, reproducibility and interrater agreement are reportedly variable. ${ }^{11,20-22}$ The insensitivity of NCCT to initial water shifts, primarily those from the interstitial to the intracellular compartment preceding progressive vasogenic edema, may preclude accurate estimation of neuronal injury in the very early aftermath of infarction. ${ }^{14,20-23}$ NCCT-ASPECTS may furthermore be limited by its tendency to cluster largely variable volumes of injury across its coarsely changing scale, as illustrated in Fig 2, in which identical NCCTASPECTS between 2 subjects can belie considerable differences in the actual volume of injury. Such challenges may underlie existing reports of greater agreement and predictive accuracy for CTP in comparison with NCCT-ASPECTS. ${ }^{13,14,24,25}$ Notwithstanding these features, we previously reported a high interrater agreement in the assignment of dichotomized ASPECTS of $>7$, despite more marginal agreement across all ASPECTSs.

We selected an infarction core threshold of $50 \mathrm{~mL}$ as a reference volume against which NCCT-ASPECTS and collateral scores were studied, as reported by the investigators of the recent SWIFT PRIME trial. ${ }^{5}$ The $50-\mathrm{mL}$ threshold has furthermore been pro-

\section{PATIENT 1}

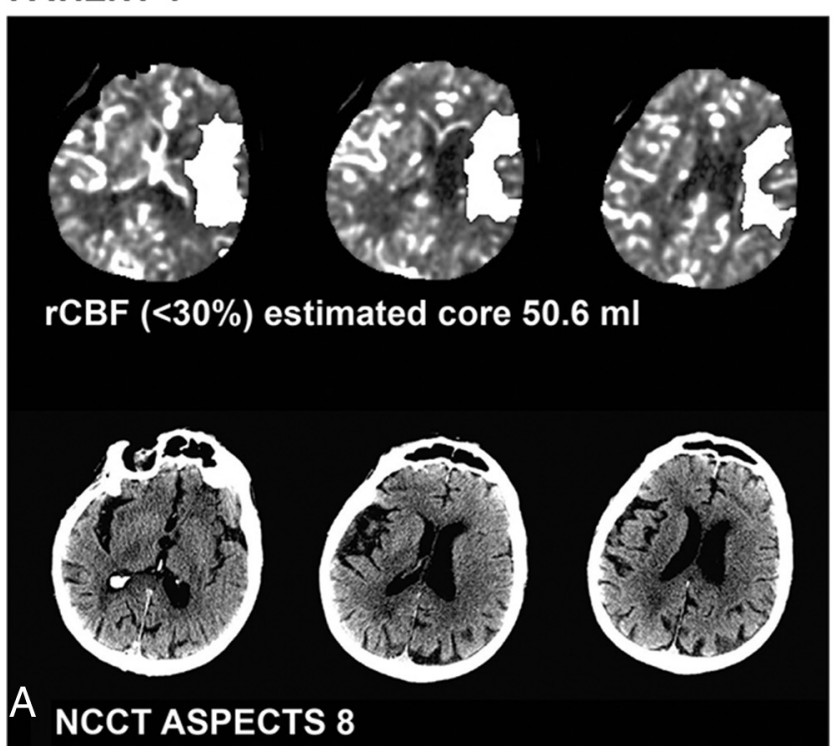

\section{PATIENT 2}

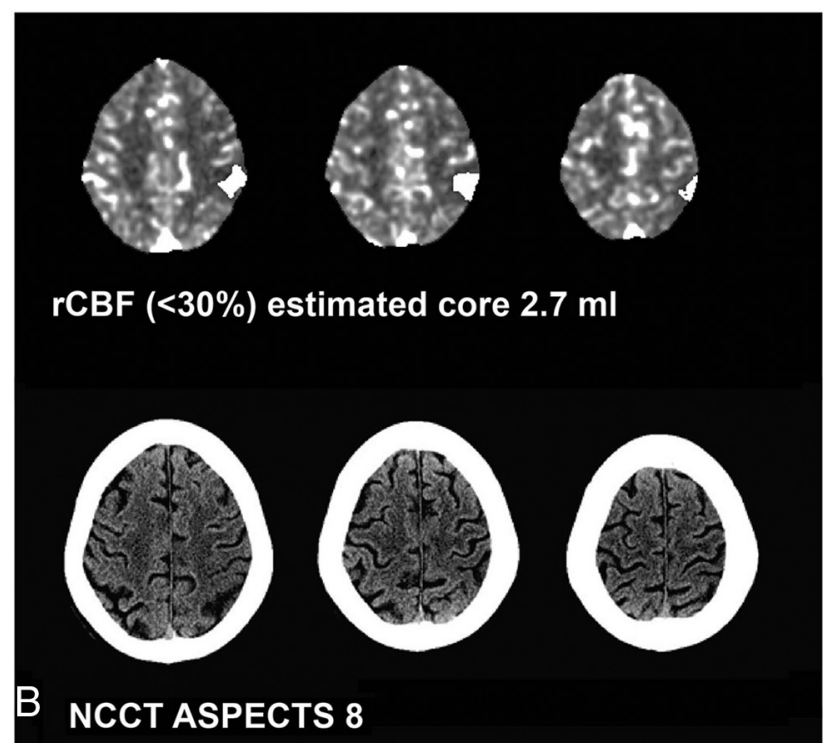

FIG 2. CT perfusion ischemic core estimates and complementary NCCT-ASPECTS in 2 patients $(A$ and $B)$. Selected images from panels of RAPID-derived CBF core maps (white overlays) and NCCT-ASPECTS in 2 subjects, both with an ASPECTS of 8 . Large differences in the estimated volume of irreversible ischemic core are noted despite high ASPECTS in both patients presenting with acute stroke-like symptoms. Patient 1 is an 83-year-old woman (NIHSS score = 29) with NCCT-ASPECTS hypoattenuation suspected within the anterior left insular region and lateral lentiform; Patient 2 is an 83-year-old man (NIHSS = 28) with NCCT-ASPECTS abnormality suspected within the lateral perirolandic parietal lobe and the lateral temporal lobe (not shown). $\mathrm{rCBF}$ indicates relative CBF. 
posed in the development of a recently reported benchmarking tool derived from pooled, prospectively acquired stroke trial data to test the accuracy of perfusion-processing software for future trial use. ${ }^{17}$ We thus propose that this threshold is relevant and reflective of current viewpoints in stroke imaging. Specifically with regard to such CTP selection criteria in AIS trials, we observed large ranges and variability in $\mathrm{CBF}$ infarction core volumes across ASPECTS. $^{5}$

Within this population, the CTA collateral score demonstrated a strong statistical association with CTP ischemic core volumes, but low sensitivity and specificity for threshold infarction prediction. While these findings could reflect statistical limitations related to sample size, we hypothesize that collateral score and other static measures of surface vascularity may be unable to capture the dynamic nature of collateral enhancement, while also lacking in their ability to identify the truly nutritive capacity of surface vessels. Recent advances in CT angiography, particularly the development of timing-invariant CTA derived from CTP dynamic bolus-passage source data, offer some promise in mitigating the timing sensitivity of standard CTA in identifying collateral vessels. $^{26}$

We acknowledge several study limitations, particularly those inherent in the retrospective nature of the analysis. Heterogeneity in the study cohort precluded subselection of treated-versus-untreated patients. However, we contend that bias related to treatment selection had a negligible impact on the study conclusions because the primary aim of our study was to examine the variability between contemporaneously acquired imaging triage strategies. The qualitative parameters in this study were generated from 2 independent, experienced readers, in whom variability may bias results; however, as previously reported, interreader agreement was high across variables in this study population. ${ }^{10}$ The relative standard in this study, against which the qualitative variables were compared, was the RAPID software environment. While other such software solutions are available, we recently reported the strengths of the RAPID tool as a fully automated, user- and vendorindependent means of semi-quantitative perfusion analysis. As a semi-quantitative CTP computing tool, RAPID has been shown to perform well, matching or exceeding the accuracy of similar software environments relative to a ground truth digital perfusion phantom in a recent study, and the use of similar iterations of the RAPID tool in recent multicenter trials may further support the generalizability of our findings. $3,4,5,7,27$

Patient selection criteria likely modulate success in achieving a favorable clinical response following revascularization in acute ischemic stroke. The era of contemporary revascularization technologies now permits timely and dependable restoration of flow in most cases; however, optimal identification of a target population for treatment remains critical, and the ideal selection strategy remains inconclusively established. These findings suggest that readily available and expedited approaches to selection such as ASPECTS correlate with commonly used perfusion parameters but may lack sensitivity to inform accurate and quantitative estimations of core volumes.

\section{CONCLUSIONS}

Using an NCCT-ASPECTS of $\geq 9$ or a CTA collateral score of 3 best predicts a $\mathrm{CBF}$ core volume infarct of $\leq 50 \mathrm{~mL}$, while an NCCT-ASPECTS of $\leq 6$ best predicts a CBF core volume infarct of $>50 \mathrm{~mL}$. Together these thresholds suggest that a specific population of patients with AIS not meeting such profiles may benefit most from CT perfusion to determine their candidacy for revascularization.

Disclosures: Matus Straka—UNRELATED: Employment: iSchemaView; Stock/Stock Options: iSchemaView. Srikant Rangaraju-UNRELATED: Grants/Grants Pending: National Institutes of Health, ${ }^{*}$ Comments: Dr Rangaraju is a trainee under the $\mathrm{Na}$ tional Institute of Neurological Disorders and Stroke T32 training grant (2T32 NS 007480-15, Principal Investigator, Allan I. Levey). *Money paid to the institution.

\section{REFERENCES}

1. Saver JL, Jahan R, Levy EI, et al. Solitaire flow restoration device versus the Merci retriever in patients with acute ischaemic stroke (SWIFT): a randomised, parallel-group, non-inferiority trial. Lancet 2012;380:1241-49 CrossRef Medline

2. Nogueira RG, Lutsep HL, Gupta R, et al. Trevo versus Merci retrievers for thrombectomy revascularisation of large vessel occlusions in acute ischaemic stroke (TREVO 2): a randomised trial. Lancet 2012;380:1231-40 CrossRef Medline

3. Lansberg MG, Straka M, Kemp S, et al; DEFUSE 2 study investigators. MRI profile and response to endovascular reperfusion after stroke (DEFUSE 2): a prospective cohort study. Lancet Neurol 2012;11: 860-67 CrossRef Medline

4. Albers GW, Thijs VN, Wechsler L, et al; DEFUSE Investigators. Magnetic resonance imaging profiles predict clinical response to early reperfusion: the diffusion and perfusion imaging evaluation for understanding stroke evolution (DEFUSE) study. Ann Neurol 2006; 60:508-17 CrossRef Medline

5. Saver JL, Goyal M, Bonafe A, et al; SWIFT PRIME Investigators. Stent-retriever thrombectomy after intravenous t-PA vs. t-PA alone in stroke. N Engl J Med 2015;372:2285-95 CrossRef Medline

6. Goyal M, Demchuk AM, Menon BK, et al; ESCAPE Trial Investigators. Randomized assessment of rapid endovascular treatment of ischemic stroke. N Engl J Med 2015;372:1019-30 CrossRef Medline

7. Campbell BC, Mitchell PJ, Kleinig TJ, et al; EXTEND-IA Investigators. Endovascular therapy for ischemic stroke with perfusion-imaging selection. $N$ Engl J Med 2015;372:1009-18 CrossRef Medline

8. Berkhemer OA, Fransen PS, Beumer D, et al. A randomized trial of intraarterial treatment for acute ischemic stroke. N Engl J Med 2015; 372:11-20 CrossRef Medline

9. Powers WJ, Derdeyn CP, Biller J, et al; American Heart Association Stroke Council. 2015 American Heart Association/American Stroke Association Focused Update of the 2013 Guidelines for the Early Management of Patients With Acute Ischemic Stroke Regarding Endovascular Treatment: A Guideline for Healthcare Professionals From the American Heart Association/American Stroke Association. Stroke 2015;46:3020-35 CrossRef Medline

10. Dehkharghani S, Bammer R, Straka M, et al. Performance and predictive value of a user-independent platform for CT perfusion analysis: threshold-derived automated systems outperform examiner-driven approaches in outcome prediction of acute ischemic stroke. AJNR Am J Neuroradiol 2015;36:1419-25 CrossRef Medline

11. Barber PA, Demchuk AM, Zhang J, et al. Validity and reliability of a quantitative computed tomography score in predicting outcome of hyperacute stroke before thrombolytic therapy: ASPECTS Study Group-Alberta Stroke Programme Early CT Score. Lancet 2000; 355:1670-74 CrossRef Medline

12. Tan IY, Demchuk AM, Hopyan J, et al. CT angiography clot burden score and collateral score: correlation with clinical and radiologic outcomes in acute middle cerebral artery infarct. AJNR Am J Neuroradiol 2009;30:525-31 CrossRef Medline 
13. Sillanpaa N, Saarinen JT, Rusanen H, et al. The clot burden score, the Boston Acute Stroke Imaging Scale, the cerebral blood volume ASPECTS, and two novel imaging parameters in the prediction of clinical outcome of ischemic stroke patients receiving intravenous thrombolytic therapy. Neuroradiology 2012;54:663-72 CrossRef Medline

14. Sillanpaa N, Saarinen JT, Rusanen H, et al. CT perfusion ASPECTS in the evaluation of acute ischemic stroke: thrombolytic therapy perspective. Cerebrovasc Dis Extra 2011;1:6-16 CrossRef Medline

15. Straka M, Albers GW, Bammer R. Real-time diffusion-perfusion mismatch analysis in acute stroke. J Magn Reson Imaging 2010;32: 1024-37 CrossRef Medline

16. Campbell BC, Christensen S, Levi CR, et al. Cerebral blood flow is the optimal CT perfusion parameter for assessing infarct core. Stroke 2011;42:3435-40 CrossRef Medline

17. Cereda CW, Christensen S, Campbell BC, et al. A benchmarking tool to evaluate computer tomography perfusion infarct core predictions against a DWI standard. J Cereb Blood Flow Metab 2015 Oct 19. [Epub ahead of print] CrossRef Medline

18. Davis SM, Donnan GA, Parsons MW, et al; EPITHET Investigators. Effects of alteplase beyond $3 \mathrm{~h}$ after stroke in the Echoplanar Imaging Thrombolytic Evaluation Trial (EPITHET): a placebo-controlled randomised trial. Lancet Neurol 2008;7:299-309 CrossRef Medline

19. Wintermark M, Albers GW, Broderick JP, et al. Acute stroke imaging research roadmap II. Stroke 2013;44:2628-39 CrossRef Medline

20. Weir NU, Pexman JH, Hill MD, et al. How well does ASPECTS predict the outcome of acute stroke treated with IV tPA? Neurology 2006;67:516-18 CrossRef Medline
21. Puetz V, Dzialowski I, Hill MD, et al. The Alberta Stroke Program Early CT Score in clinical practice: what have we learned? Int J Stroke 2009;4:354-64 CrossRef Medline

22. Dzialowski I, Hill MD, Coutts SB, et al. Extent of early ischemic changes on computed tomography (CT) before thrombolysis: prognostic value of the Alberta Stroke Program Early CT Score in ECASS II. Stroke 2006;37:973-78 CrossRef Medline

23. Aviv RI, Mandelcorn J, Chakraborty S, et al. Alberta Stroke Program Early CT Scoring of CT perfusion in early stroke visualization and assessment. AJNR Am J Neuroradiol 2007;28:1975-80 CrossRef Medline

24. Lin K, Rapalino O, Law M, et al. Accuracy of the Alberta Stroke Program Early CT Score during the first 3 hours of middle cerebral artery stroke: comparison of noncontrast CT, CT angiography source images, and CT perfusion. AJNR Am J Neuroradiol 2008;29: 931-36 CrossRef Medline

25. van Seeters T, Biessels GJ, Niesten JM, et al; Dust Investigators. Reliability of visual assessment of non-contrast CT, CT angiography source images and CT perfusion in patients with suspected ischemic stroke. PLoS One 2013;8:e75615 CrossRef Medline

26. Smit EJ, Vonken EJ, van der Schaaf IC, et al. Timing-invariant reconstruction for deriving high-quality CT angiographic data from cerebral CT perfusion data. Radiology 2012;263:216-25 CrossRef Medline

27. Kudo K, Christensen S, Sasaki M, et al; Stroke Imaging Repository (STIR) Investigators. Accuracy and reliability assessment of CT and MR perfusion analysis software using a digital phantom. Radiology 2013;267:201-11 CrossRef Medline 